- Dental implant placement must be both biologically and restoratively driven.

- Dental implants should only be placed following a comprehensive examination and accurate diagnosis.

- The deficient osseous ridge must be reconstructed prior to implant placement.

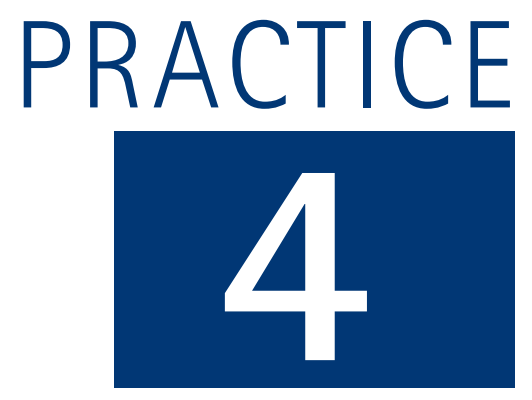

\title{
Surgical guidelines for dental implant placement
}

\author{
M. Handelsman ${ }^{1}$
}

\begin{abstract}
The goal of an implant supported reconstruction is to obtain optimal aesthetics and function. In order to achieve this, visualisation of the final restorative reconstruction is necessary prior to beginning treatment. The term 'restorative-driven' treatment planning has been used to identify this process. ${ }^{1}$ It requires a team approach of specialists, who can develop a multidisciplinary treatment plan. It starts with an accurate diagnosis, which will lead to a prognosis of each individual tooth and the overall dentition. This information will help the clinician develop the treatment options suitable for tooth replacement. Only when the goals have been defined can the sequence of therapy be established. Working backwards from the wax-up of the final diagnostic model of the proposed treatment assists not only with the management of the complex case, but will help avoid mishaps. ${ }^{2}$ Effective communication between the team and the patient is extremely important. Understanding the patient's expectations is key to a successful outcome. Deciding that these expectations are realistic requires a correct diagnosis and an inter-disciplinary treatment plan that is logical. This approach takes time and requires a comprehensive treatment discussion between the team members, and then a thorough case presentation to the patient. ${ }^{3}$ Only then will the patient begin to understand the extent of their problem and the options available to reconstruct their mouth.
\end{abstract}

\begin{tabular}{|c|}
\hline IMPLANTS \\
\hline 1. Rationale for dental implants \\
\hline $\begin{array}{l}\text { 2. Treatment planning of implants in } \\
\text { posterior quadrants }\end{array}$ \\
\hline $\begin{array}{l}\text { 3. Treatment planning of implants in } \\
\text { the aesthetic zone }\end{array}$ \\
\hline $\begin{array}{l}\text { 4. Surgical guidelines for dental } \\
\text { implant placement }\end{array}$ \\
\hline $\begin{array}{l}\text { 5. Immediate implant placement: } \\
\text { treatment planning and surgical } \\
\text { steps for successful outcomes }\end{array}$ \\
\hline $\begin{array}{l}\text { 6. Treatment planning of the } \\
\text { edentulous maxilla }\end{array}$ \\
\hline $\begin{array}{l}\text { 7. Treatment planning of the } \\
\text { edentulous mandible }\end{array}$ \\
\hline $\begin{array}{l}\text { 8. Impressions techniques for } \\
\text { implant dentistry }\end{array}$ \\
\hline $\begin{array}{l}\text { 9. Screw versus cemented implant } \\
\text { supported restorations }\end{array}$ \\
\hline $\begin{array}{l}\text { 10. Designing abutments for } \\
\text { cement retained implant supported } \\
\text { restorations }\end{array}$ \\
\hline 11. Connecting implants to teeth \\
\hline $\begin{array}{l}\text { 12. Transitioning a patient from } \\
\text { teeth to implants }\end{array}$ \\
\hline $\begin{array}{l}\text { 13. The role of orthodontics in } \\
\text { implant dentistry }\end{array}$ \\
\hline $\begin{array}{l}\text { 14. Interdisciplinary approach to } \\
\text { implant dentistry }\end{array}$ \\
\hline $\begin{array}{l}\text { 15. Factors that affect individual } \\
\text { tooth prognosis and choices in } \\
\text { contemporary treatment planning }\end{array}$ \\
\hline 16. Maintenance and failures \\
\hline
\end{tabular}

\section{DIAGNOSIS}

The restorative dentist will develop and direct the plan after gathering data, which includes a complete medical and dental history, clinical findings, photographs, mounted diagnostic casts and radiographs. Consultation with other specialists (periodontist, oral surgeon, orthodontist, endodontist) regarding the periodontal and endodontic health, and any occlusal, skeletal and space problems will be required.

A correct diagnosis with long-term prognostic information is mandatory, in order to develop an interdisciplinary treatment plan. All the treating specialists on the team need to collaborate their findings, which includes the following examinations.

\section{A. Periodontal examination (Figs 1-8)}

A comprehensive periodontal examination includes the soft and hard supporting tissues of the dentition. The patient's tissue biotype is classified according to how thick or thin the supporting bone and gingival soft tissues are defined. Becker and Oschenbein ${ }^{4}$ classified three distinct

${ }^{1}$ Diplomate: American Board of Periodontology / Private Practice: Santa Monica, California

Correspondence to: Dr Mark Handelsman, 1245 Sixteenth Street, \#206, Santa Monica, CA 90404, USA

Email: markhandelsman@aol.com

\section{Refereed Paper}

( $)$ British Dental Journal 2006; 201: 139-152

DOI: $10.1038 /$ sj.bdj.4813820 types: pronounced scalloped, scalloped and flat. A thin periodontium will be pronounced scalloped or scalloped. A thick periodontium will present with flat gingival architecture, usually supported by thick buccal and lingual plates of alveolar bone. A thin skeletal pattern with scalloped architecture will have root dehiscence and fenestrations even in a healthy periodontium. If periodontal disease is present, it is important to establish the attachment level (probing depths and gingival recession) as well as any contributing factors, such as mucogingival problems (lack of keratinised attached tissue) and furcation involvement, which will alter the prognosis of the remaining teeth. It is critical to measure not only probing depths, but identify the level of the crestal alveolar bone. Kois ${ }^{5}$ classified a high crest when the crestal bone level is close to the CEJ (delayed passive eruption). The normal crest is defined as $2 \mathrm{~mm}$ from the CEJ and the low crest is present in patients with recession. Sounding to bone is the best clinical parameter to help identify the attachment level. This is extremely important in the aesthetic zone, when considering replacing partially edentulous teeth. The interproximal attachment level on the teeth adjacent to a future implant site will dictate the future gingival architecture. ${ }^{6}$ In the aesthetic zone it is extremely important to identify the amount of teeth and soft tissues visible not only from the frontal view, but also from the lateral view, both with the lip at rest 
and also when smiling. Uneven gingival architecture, the position of teeth relative to the arch shape and opposing occlusion will all affect and dictate the decision making process.

The presence of bone loss due to periodontal disease or trauma will greatly affect the outcome of treatment. If teeth are already missing then the amount of ridge collapse needs to be measured according to both horizontal and vertical collapse. (Siebert Classification type I, II and III.) Lekholm and Zarb ${ }^{7}$ classified the hard tissue according to the shape of the ridge (morphology) and the bone quality; shape a-e and type 1-4 respectively. This will be discussed in greater detail later in the article as relating to surgical technique.

If the prognosis of teeth is deemed hopeless due to periodontal, endodontic or non-restorability factors, then anticipating the amount of future ridge collapse needs to be estimated. In this case, it is extremely important to identify the tissue biotype and the underlining missing bone, which will help determine the prognosis and steps necessary to preserve or rebuild the desired soft and hard tissue architecture after tooth loss. Thick tissue is much more forgiving, easier to manipulate and provides a more predictable aesthetic outcome, as compared to thin tissue which is more likely to shrink. Deciding when to extract a tooth is easy when it is hopeless, but often more heroic attempts to save teeth that are broken down with a questionable prognosis is not as simple. In order to preserve the alveolar bone for implants, sacrificing teeth (early extraction) requires a change of thinking as compared to previous philosophical treatment decisions.

\section{B. Occlusal analysis}

Patients with occlusal wear or abfraction type defects due to clenching or bruxism should be identified. The parafunctional habits will greatly affect the outcome and longevity of the type of reconstruction planned. The opposing occlusion along with the type of restorative materials selected for the final prosthesis will affect the ability of the bone-implant interface to withstand the occlusal load. In the periodontally compromised patient, loss of teeth without replacement leads to lack of posterior support. This often causes an unstable occlusal scheme with mesial drifting of posterior teeth and flaring of anterior teeth with a loss of vertical dimension. A full examination includes not only the mobility of remaining teeth, but also the occlusal scheme and guidance in lateral and protrusive movements. Over-eruption of any teeth will cause occlusal interferences and also decrease the inter-occlusal space necessary for implant restorative components. Lack of restorative space is a major contributor to mechanical failure of implant restorations. Mounted diagnostic casts are used to evaluate the occlusion, the edentulous space ridge relationship to the adjacent teeth and the opposing dentition. The amount of vertical and horizontal over- lap as well as the restorative space available is extremely important. Replacing teeth in partially edentulous spaces, without comprehensive care of the remaining dentition, is sure to cause failure in the long term.

\section{Endodontic}

The endodontic integrity and vitality of remaining dentition must be established. Any pathological changes, such as periapical lesions and existing root canals that are incomplete need to be evaluated. Any teeth with a poor endodontic prognosis should have a thorough risk assessment completed, so as not to jeopardise future implant sites.

\section{Restorative}

The integrity of all existing restorations should be evaluated. This must include margin integrity and soft tissue response to sub-gingival placement. Teeth with biologic width violation, that will require surgical crown lengthening, will affect the future level of the osseous crest at sites adjacent to implants. In the aesthetic zone, it is important to anticipate the future gingival contours of the teeth adjacent to the implant site. Teeth that are fractured, broken down beyond predictable restoration, or have compromised support will be given a poor or hopeless prognosis. The strategic value of each tooth needs to be determined, prior to removal. Often in complex cases, even teeth with a poor prognosis can be kept in the initial phases to support a fixed interim prosthesis, while implant healing progresses. This phased approach of sequential extraction will help manage the complex case, sometimes through long periods (two years) while site development and healing of implant sites is progressing.

\section{E. Orthodontic}

Analysis of the restorative space is important regarding future tooth restoration size, but it is equally important to analyse the position and angulation of the roots in the alveolar bone. Drifting and tipping of remaining teeth will often present problems with space mesially-distally. The orthodontist needs to be given clear instructions when opening or closing spaces in regards to root positions. Taking radiographs during treatment will give useful information to the treating team, prior to appliance removal and completion of treatment. It is always preferable in aesthetic cases to complete the orthodontic care prior to implant placement. In cases missing posterior teeth, provisional interim implants can be placed to help the orthodontist establish anchorage. The final implants should only be placed posteriorly, if the orthodontic wax-up of the final result allows for correct positioning, without compromising the end result.

\section{F. Radiographic examination. (Figs 28-33)}

The preliminary exam will include a set of full mouth periapical and bitewing radiographs. A 
panorex is helpful as well, but limited compared to 3-D CT scan imaging. Once the patient has had the initial consultation and accepted the proposed options, a diagnostic wax-up of the final proposed treatment will be a template to fabricate a radiographic guide. This radiographic guide can be placed in the mouth at the time of the CT scan. Transferring the restorative template on to the radiographic images is the most useful information for determining the surgical plan. Lack of bone and ridge collapse both horizontally and vertically can be accurately measured. The surgical needs can be identified and the most predictable options can be presented to the patient prior to beginning treatment. The goals of the final reconstruction, whether fixed or removable, can be determined. The treatment expectations can be presented to the patient, with realistic steps of what can and cannot be accomplished. Only then can the treatment plan in a phased approach, be determined by all the treating doctors on the team. All financial obligations, treatment consent as well as aesthetic goals are established prior to taking any further steps.

Occasionally, when questionable teeth are still present, it may be beneficial to have a CT scan prior to removing teeth. This can help make treatment decisions regarding surgical options of timing of implant placement (see discussion later in article).

\section{Pre-surgical planning}

Viewing the CT images which have the crosssectional reformatted images of the bone quantity and quality at each desired implant site, allows the surgeon to develop the surgical plan. The question most often asked - 'Do I have enough bone?' - can be answered with an educated answer. The goals of the restorative dentist can be accomplished or cannot be achieved. In complex fully edentulous cases, whether fixed or removable reconstruction will be the best outcome can be determined. The surgeon can then modify the radiographic guide to be used as a surgical guide on the day of surgery. Recent developments in CAD-CAM technology allow the team to perform pre-surgical planning on a virtual model and then transfer this information to a clinical surgical guide to assist accurate placement of implants (eg Nobel Biocare ARK, Simplant).

The hard tissue classification by Lekholm and Zarb defined quantity and quality of the edentulous ridge.

\section{Quality}

Type 1: Almost the entire jaw is composed of homogenous compact bone.

Type 2: A thick layer of compact bone surrounds a core of dense trabecular bone.

Type 3: A thin layer of cortical bone surrounds a core of dense trabecular bone of favourable strength.

Type 4: A thin layer of cortical bone surrounds a core of low density trabecular bone.

\section{Quantity: Ridge shape a-e.}

Shape 'a' represents no bone resorption, while 'e' represents advanced ridge resorption.

The pattern of resorption in the anterior maxilla (Figs 5-8) advances posteriorly and superiorly, which greatly affects the aesthetic zone and lip support. The pattern of resorption of the posterior ridges greatly affects the occlusion and horizontal overlap of the dentition ${ }^{8}$ (Figs 9-13).

\section{SURGICAL GUIDELINES - TOOTH OR TEETH ALREADY MISSING}

\section{Adequate osseous ridge}

The Brånemark surgical protocol ${ }^{9}$ established osseointegration as an extremely predictable option for tooth replacement with excellent long term stability. Many implant systems have since been developed which use this concept. The numerous shapes and sizes of implants available for use on patients today continue to change and are overwhelming. It is extremely important for all members of the team to discuss which components are to be used surgically and restoratively, so that the benefits and limitations are defined. Whether the prosthesis will be screw retained or cement retained can affect the axial inclination and angle of placement. Due to common anatomical limitations post-extraction (concavities and collapse of the ridge circumference), using cement retained restorations allows greater flexibility at the time of surgical placement.

\section{Flap design}

The original Brånemark protocol required a vestibular flap with a two stage approach. The implant was placed and buried under the soft tissue and after an adequate period of healing (three months mandible, six months maxilla), a second stage surgery was performed with crestal incisions to expose the fixtures and connect a trans-epithelial abutment. After adequate soft tissue healing, the restorative dentist could fabricate the prosthesis. One stage surgical protocols were developed by ITI in Switzerland ${ }^{10}$ that allowed the implant fixture to extend through the soft tissues during the period of initial healing. This protocol was duplicated and shown to be effective using a two stage system with the same predictability. ${ }^{11}$ Today this trend continues with most implants placed with a one stage protocol allowing adequate time for healing prior to loading the fixtures. Using the one stage technique requires a crestal incision or even a flapless approach, drilling directly through the soft tissues. Obviously, deciding which cases are suitable for the flapless technique is the key to a successful outcome; adequate bone width with plenty of keratinised soft tissue is desired. Compromising the site while drilling due to lack of visibility is not advised. In the aesthetic zone it is important to maintain adequate thickness on the buccal aspect of the implant for long term stability of the soft tissue contours. Usually this area lacks enough tissue due to resorption subsequent to tooth loss, and augmentation techniques are required. The L. Abram's roll 


\section{PRACTICE}

Fig. 1 (left) Anterior X-section of thick periodontium with high crest (bone at level of CEJ)

Fig. 2 (right) Thin periodontium with low crest (Note the bone dehiscence on the buccal surface)

Fig. 3 (left) Periodontitis with advanced bone loss and probing depths; expect vertical ridge collapse after tooth extracted

Fig. 4 (right) Recession; attachment loss with advanced horizontal bone loss; expect even larger ridge defect after tooth loss

Fig. 5 (left) Type 'a' ridge (thick periodontium) with no collapse

Fig. 6 (right) Loss of vertical height after extraction of teeth with

moderate to advanced periodontitis (normal to thick periodontium). This will also present with loss of papilla height, due to previous interproximal bone defect

Fig. 7 (left) Expected collapse of ridge after extraction with thin periodontium (even in healthy environment)

Fig. 8 (right) Shape 'c' or 'd'. Thin periodontium with pronounced scalloped biotype and disease (see Figs 3-4) will usually shrink more, leaving concavity type defect with knife edge ridge

Fig. 9 (left) Posterior x-section showing horizontal overlap and ridge relationship in health

Fig. 10 (right) Tooth loss with minimal ridge collapse. (Type 'a' and 'b' ridge shape)
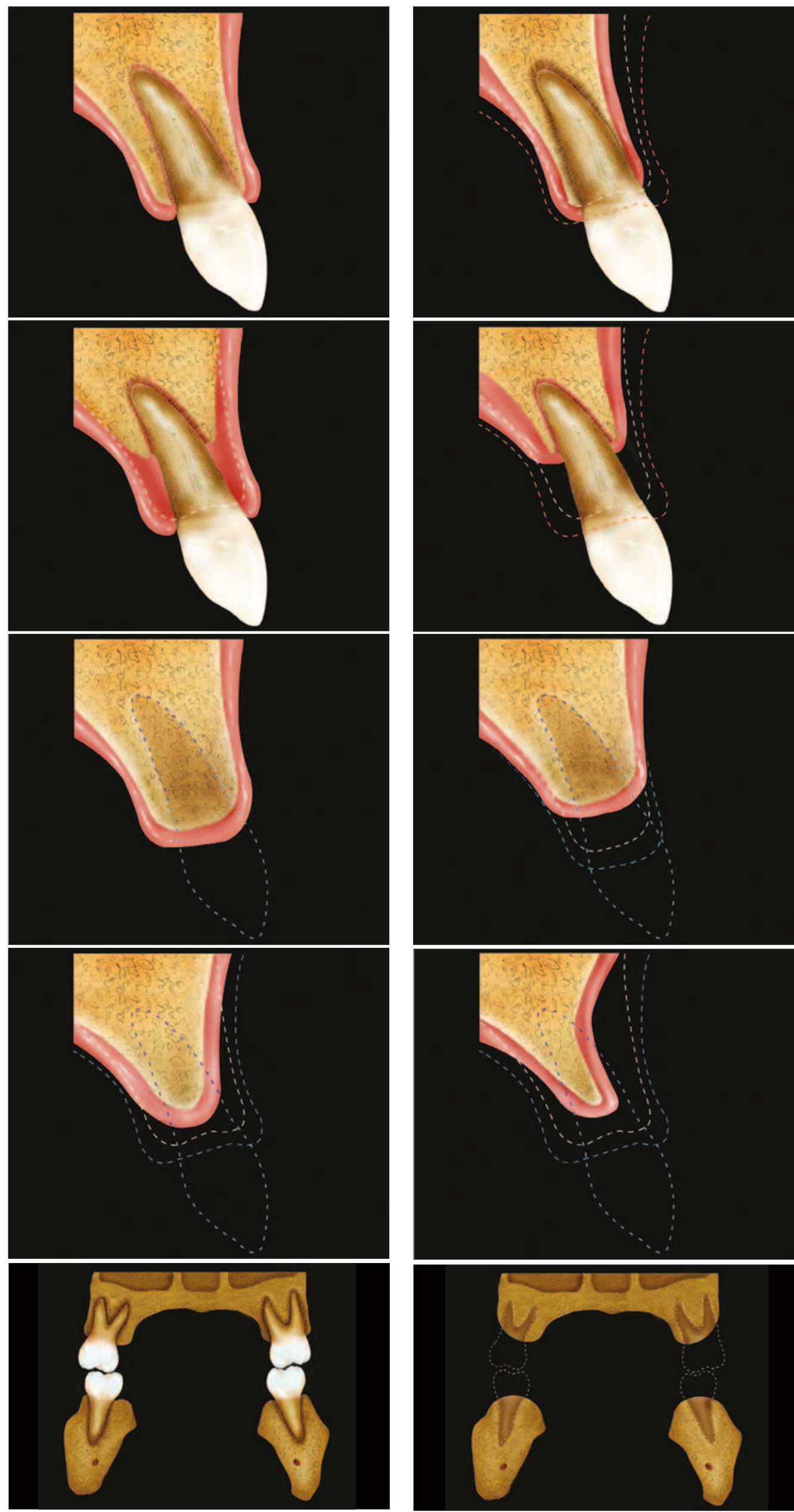

BRITISH DENTAL JOURNAL VOLUME 201 NO. 3 AUG 122006 

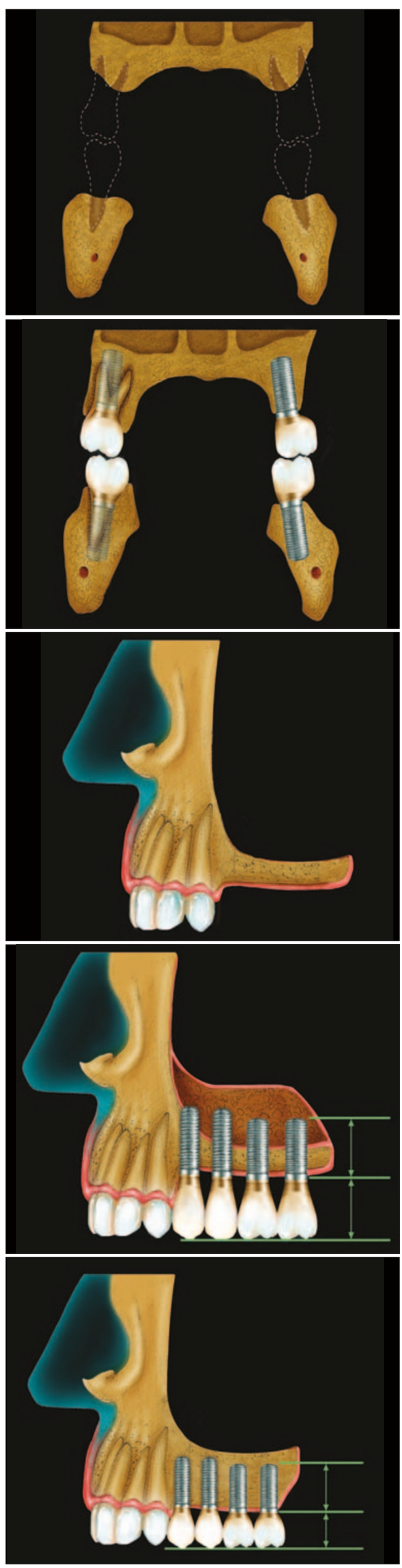
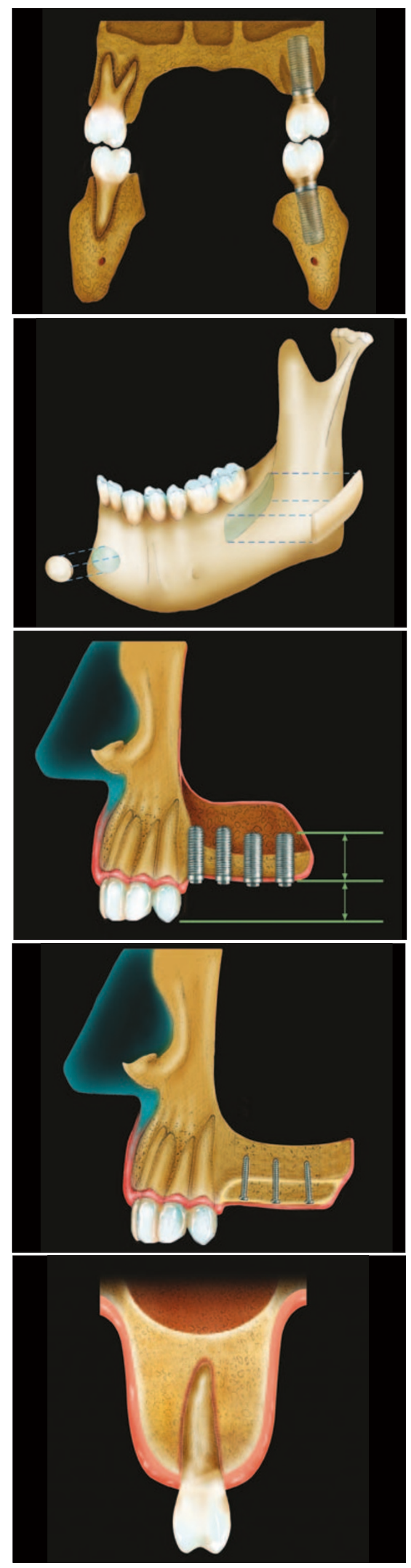

Fig. 11 (left) Further resorption creates greater interarch distance with less bone available to place only shorter implants

Fig. 12 (right) Minimal resorption allows occlusal scheme to remain the same as prior to extraction, and ideal emergence for crown contours

Fig. 13 (left) Buccal collapse of the ridges (left) requires implants to be placed in a more lingual position. This creates restorations with buccal overcontouring and unfavourable loading conditions on the implant. The alternative option is to restore with an $\mathrm{x}$-bite relationship. Note on the other side of diagram, short clinical crowns require countersinking of the implants to allow adequate space for restorative components

Fig. 14 (right) Intra-oral graft sites include ramus and chin

Fig. 15 (left) Posterior maxilla presents with enlarged sinus and not enough bone height to place implants. Note minimal amount of vertical ridge height collapse

Fig. 16 (right) Sinus Lift using window approach through the lateral wall. After adequate bone healing (four to six months), implants are then placed. Simultaneous bone grafting and implant placement is a risker procedure. Note crown to implant ratio will be favourable

Fig. 17 (left) Note more advanced ridge resorption. Sinus Lift without reconstruction of ridge height leads to less favourable crown to implant ratio. Placement of the most mesial implant adjacent to the cuspid tooth on the incline creates problem with Interproximal tissues and forces implant placement slightly more distal than ideal in order to protect attachment on distal of cuspid

Fig. 18 (right) Onlay block graft with fixation screws to reconstruct the ridge height and improve crown contours

Fig. 19 (left) After adequate healing, screws are removed and implants are placed. Note ideal crown/implant ratio

Fig. 20 (right) Posterior maxilla with ideal bone height and width 
technique $^{12}$ is often used to transfer the crestal thicker tissue onto the buccal aspect, rather than using a punch technique (flapless approach) which is subtractive.

Using a two stage protocol in the aesthetic zone also allows for an additional opportunity for surgical intervention with further hard or soft tissue grafting procedures. Langer ${ }^{13}$ introduced the sub-epithelial connective tissue graft as a predictable technique for augmenting thin tissue and it is especially useful when minor ridge resorption is present. The most common donor site is the palatal tissue mesial to the first molar. Another popular donor site is the tuberosity tissue. This area is especially desirable when a thicker graft is needed for ridge augmentation procedures (inlay or pouch technique) ${ }^{14}$ in the anterior aesthetic zone. Palacci ${ }^{15}$ has also described rotated flap techniques for papilla regeneration. It is always important to define the type of defect, and realise that building the supporting bone beneath the soft tissue will produce the most predictable outcome. The bony architecture provides the scaffold that supports the soft tissue around proper implant positioning.

\section{POSITION AND ANGULATION OF IMPLANT}

Presurgical analysis has determined the best available sites for placement. In the partially edentulous case, being aware of the root morphology and inclination of teeth adjacent to the implant site is crucial. If a radiographic guide was used at the time of CT scan and then adapted for use as a surgical guide, mishaps will be prevented. If a surgical guide is fabricated without the knowledge of the root positions or bony deficiencies, then careful interpretation and adjustments by the surgeon is required. The initial drilling (round bur) starting point can be moved and the angulation of the implant tipped to avoid bone dehiscence or fenestration. The more palatal the movement, the deeper sub-gingivally the implant platform must be positioned to accommodate for the emergence of the restorative components through the soft tissue. Ideal dimensions ${ }^{16}$ are $3-4 \mathrm{~mm}$ subgingival, with the buccal aspect of the implant platform at least 1 $\mathrm{mm}$ or more palatal or lingual to the future buccal aspect of the restoration at the level of the gingival margin. Placing a wider platform in the anterior aesthetic region provides for a smoother gingival restorative interface (platform does not have to be as deep), but invites greater risk of apical migration of the soft tissue margin. The concept of 'platform switching' - using a narrower restorative component on a wider implant platform (possible only with some implant systems, eg 3i) - is interesting. ${ }^{17}$ It appears to help support the soft tissue and prevent crestal resorption of the bone at the level of the implant platform. At this time further research is needed.

\section{Dense bone}

Dense bone (Type 1 \&t 2 ) requires careful attention to drilling with adequate irrigation in order not to overheat the bone. Care should be taken to debride the osteotomy site from bone debris and to widen the site to the manufacturer's recommended diameter prior to tapping the site. Placing the implant into too tight a site can lead to failure due to pressure necrosis. The shape of the implant, parallel walled vs. tapered will also greatly affect the tightness (primary stability).

\section{Softer bone}

Type 3 and 4 bone requires modification of the drilling protocol. Care must be taken to underprepare the osteotomy site. Over-preparation or inadvertent implant angulation changes can preclude placement of the implant. Using the Osteotome technique ${ }^{18}$ can also help initial stabilisation by compressing the available soft bone instead of compromising the site with drilling. Tapered implants have an advantage in softer bone due to the wedging effect at the time of placement. The tapered implant is more challenging at the time of placement, because the depth of the prepared site needs to be exactly at the level of where the desired final position of the implant needs to be placed. As opposed to a parallel wall implant that can be placed deeper if desired, due to the ability to sink the implant into the prepared site. The twist drills for the parallel wall implants have markings, which prepare the depth slightly longer than the implant length. Due to this design and extra tip at the end of the drill, care should always be taken when drilling above vital structures such as the alveolar canal in the posterior mandible.

Bi-cortical fixation in softer sites helps anchor the fixture especially in the posterior maxilla. Engaging the sinus floor is suggested to achieve this anchorage.

Countersinking into the crestal bone may cause loss of initial stabilisation at the time of placement. The need to countersink the implant is usually restorative driven for aesthetic and emergence purposes or due to lack of restorative space. The original protocol required countersinking to protect the implant from trans-mucosal forces generated by removable appliances over the implant head. In softer bone, it is preferable to place the platform of the implant at the crest or supra-crestal if space allows for adequate emergence.

\section{The deficient osseous ridge (Figs 34-61) Lack of vertical height}

Anterior maxilla: Vertical resorption of the ridge will affect the aesthetic result in partially edentulous cases. In fully edentulous cases the amount of loss might limit treatment to removable overdentures.

Options for surgical correction include:

- Onlay bone grafting

- Distraction osteogenesis.

The onlay block graft ${ }^{19}$ requires an autogenous block of bone be taken from either intraoral sites (Ramus or chin) (see Fig. 14) or extraoral site (iliac crest or calvaria). The greater the defect, the more bone required. Analysis of the 
dimensions required will dictate which donor site is preferable. Extra-oral sites require general anaesthesia and increase the risk of greater morbidity. Intra-oral block grafts are better suited for partially edentulous situations. The block of cortical cancellous bone is carefully adapted to the recipient site and fixated with screws to stabilise the graft during the healing period. Building vertical height is the least predictable of all the grafting options.

Distraction Osteogenesis ${ }^{20}$ allows the existing bone to be transferred to a more coronal position after surgical osteotomy cuts and placement of a device that is activated daily in the mouth. After several weeks of movement, the bone is left to mature for a few months prior to implant placement.

Posterior maxilla: The posterior maxilla has historically always been the most challenging site to achieve success. The most common site for tooth loss in the periodontally compromised patient is the maxillary molars due to furcation involvement. ${ }^{21}$ Due to a combination of periodontal bone loss and sinus proximity, limited bone height is usually available for implants (Fig. 15). The bone is also usually soft (type 3 or 4), which makes initial stabilisation difficult to achieve. Several techniques have been developed and perfected over the last decade to improve the surgical options in the posterior maxilla (Figs 16-24).

The Sinus Lift procedure ${ }^{22}$ reported by Boyne et al. showed that it is possible to open the maxillary sinus through the lateral wall, elevate the sinus membrane and pack bone grafting material into the space. Many types of bone grafting materials are available. Autogenous bone has always been the most predictable option that has set the gold standard. Intra-oral bone can be harvested from the chin, ramus or tuberosity areas. This bone is ground and the particulated graft material is best combined with an osteo-conductive material of the surgeon's choice (eg Hydroxy-apatite, Bio-oss or freeze dried bone). Using Platelet Rich Plas$\mathrm{ma}^{23}$ to aid the healing response is an additional option. It is more predictable to perform the sinus lift graft, and then wait 5-6 months for healing prior to implant placement. If $5 \mathrm{~mm}$ or more of bone height is available, simultaneous implant placement with sinus lift and bone grafting is possible, with less predictability. If 7 or $8 \mathrm{~mm}$ of height is available, then placement of the implants with an osteotome technique for increasing the length of implant is possible (see Figs 20-24). The protocol calls for drilling the initial twist drills at least $3 \mathrm{~mm}$ short of the sinus floor. The osteotome is placed into the osteotomy. The sinus floor is in-fractured, thereby raising the membrane and creating space for the graft. Bone graft material can then be pushed into the preparation site prior to placing the implant. This technique is popular, because it seems less invasive as compared to the sinus lift via the lateral wall technique, but obviously it requires careful patient selection. It is a technique sensitive procedure, good for single tooth replacement, but with less predictability, due to the greater potential for sinus membrane perforation.

Posterior mandible: The inferior alveolar nerve needs to be visualised in order not to cause nerve damage such as paraesthesia. It often limits implant placement to short wide implants. Alternative options are the nerve transposition procedure $^{24}$ which has a high morbidity and rate of nerve damage, or to consider distal cantilevering of the prosthesis off implants placed mesial to the foramina. Distraction osteogenesis can also be used if inter-arch space is not a problem.

\section{Lack of horizontal width}

Anterior maxilla: The pattern of resorption usually creates knife-edge ridges or ridges that are too thin due to concavities (Fig. 8). The anterior incisal foramen is sometimes large and can restrict implant placement in the maxillary central positions (Fig. 37).

Knife-edge ridges are best treated with veneer grafts ${ }^{19}$ using an intra-oral block of corticalcancellous autogenous bone stabilised with fixation screws (Figs 25-26). Unlike onlay grafts, these veneer grafts are more predictable. Concavity defects can be treated with block grafts, but these also respond favourably to $\mathrm{GBR}^{25}$ procedures (Fig. 27). An intra-oral block harvested from the ramus can be ground, or a scraping device (Safescraper, 3i Palm Beach, Florida, USA or Ebner 502 grafter, Maxillon Lab Inc. Hollis, NH. USA) can harvest scrapings off the ramus cortical plate which can then be mixed with an osteo-conductive material to create a particulate graft. A resorbable or non-resorbable membrane is then fixated with pins over the graft material. A titanium re-inforced membrane ${ }^{26}$ can be used if spacemaking is desired.

Narrow ridges in the maxilla can also be treated by spreading the bone with osteotomes or a more aggressive approach, the Split-Ridge technique, which widens the existing bone with simultaneous implant placement.

Anterior and posterior mandible: Block grafts with fixation screws (GBR) as described above are possible. Determining the depth of the vestibule and the ability to advance the flap for graft coverage without any tension, is the key to achieve success.

\section{SURGICAL GUIDELINES - TOOTH OR TEETH NEED TO BE REMOVED}

If the tooth or teeth need to be removed, then ask the following questions.

- Is the tooth in the aesthetic zone?

- Type of tissue biotype? Thin scalloped or thick flat.

- Amount of bone loss due to periodontal disease or endodontic failure?

- How predictable is the stability of the future gingival architecture?

There are several timing options to consider:

1. Extract and wait several months prior to implant placement. 
Fig. 21 (left) Extraction due to periodontal bone loss, along with sinus enlargement, leads to reduced height for implant placement

Fig. 22 (right) The Osteotome technique is useful when $7 \mathrm{~mm}$ or more bone height is available. Drill to within $3 \mathrm{~mm}$ of sinus floor, then start with osteotome

Fig. 23 (left) Use wider osteotome to fracture sinus floor upwards, and then pack bone graft material into osteotomy site prior to implant placement (shown in grey in this diagram)

Fig. 24 (right) Place implant into site

Fig. 25 (left) Stabilisation of intra-oral autogenous cortical block bone with fixation screws to correct knife-edge ridge with some vertical loss

Fig. 26 (right) Screws are removed after six months

Fig. 27 (left) Concavity type defects with no vertical loss can be reconstructed using Guided Bone Regeneration using particulate graft and membranes

Fig. 28 (right) Surgical Guide. This guide was adapted for use as a radiographic guide. The holes were filled with gutta-percha. The outside surface of the teeth (palatal, occlusal and buccal) were wrapped with foil. This produces the following images (Figs 29, 31, 33)

Fig. 29 (left) CT scan axial cut showing radiographic markers transferred onto images

Fig. 30 (right) Oblique cuts showing posterior maxilla pre-bone grafting. No radiographic guide at time of scan
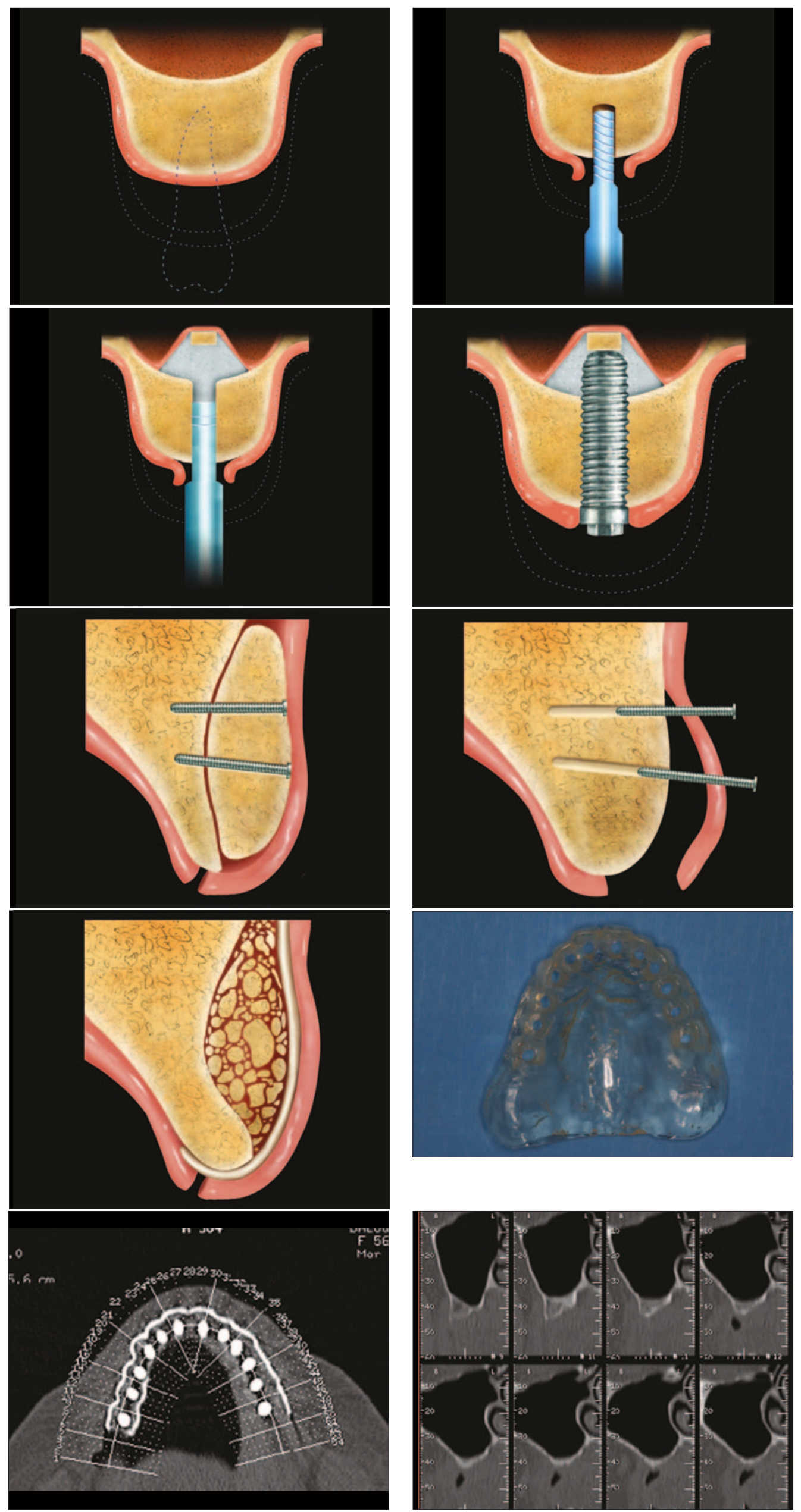

BRITISH DENTAL JOURNAL VOLUME 201 NO. 3 AUG 122006 

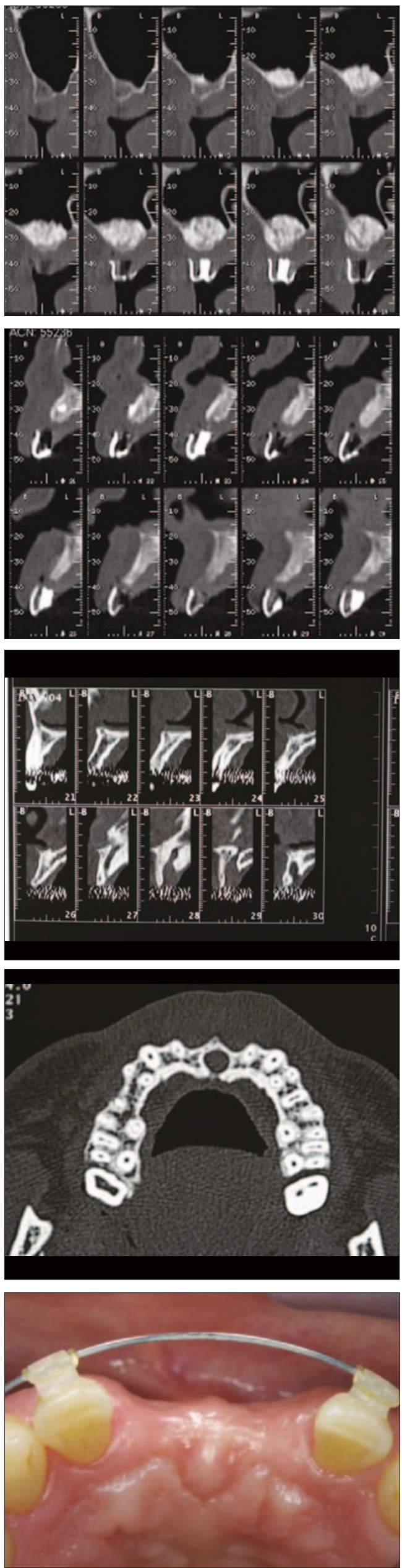
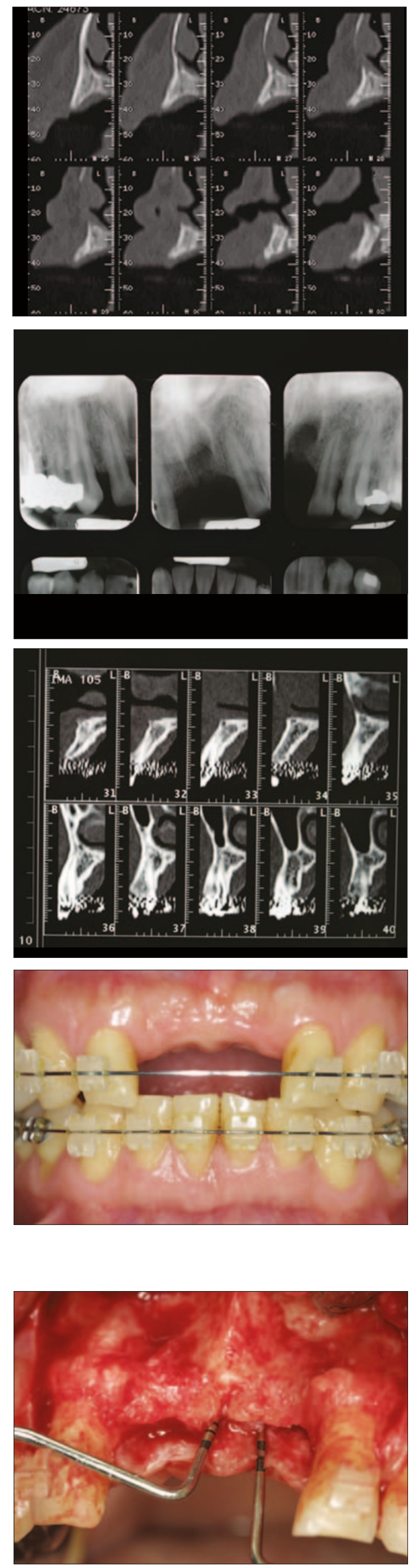

Fig. 31 (left) Oblique cuts showing posterior maxilla post - bone grafting with sinus lift procedure. Note radiographic marker shows outline of desired crown contours and internally desired implant location and angulation

Fig. 32 (right) Oblique cuts showing maxillary anterior region pre-grafting. Note no radiographic marker and knife-edge ridge

Fig. 33 (left) Oblique cuts showing anterior region post- bone grafting with intra-oral autogenous block graft buccal veneer. Note fixation screw (red arrows)

Fig. 34 (right) Patient presented with missing central incisors, diastemata and uneven occlusal plane. Initial radiographs of the maxillary anterior region

Figs 35-36 CT scan of oblique cuts showing advanced bone resorption with thin ridge and concavity defect.

The anterior incisal foramen is extremely large

Fig. 37 (left) CT scan of maxilla. The axial cut shows the problem of the large foramen which restricts implant placement

Fig. 38 (right) Clinical anterior view during orthodontic therapy to correct occlusal plane and space problems

Fig. 39 (left) Clinical occlusal view showing advanced ridge resorption

Fig. 40 (right) Flap reflection shows buccal concavity. Periodontal probes indicate location of foramen 
Fig. 41 (left) Palatal flap with neuro-vascular bundle removed from foramen

Fig. 42 (right) Harvesting autogenous block bone from lateral region of the ramus

Figs 43-44 Cortical block of bone prior to shaping

Fig. 45 Preparation of the recipient site requires drilling small holes into the cortical layer to expose bleeding sites

Fig. 46-47 Close approximation of graft to recipient site is required. Block is stabilised with fixation screws

Fig. 48 (left) Particulate bone mixed with osteo-conductive bone graft material (Hydroxy-apetite) is packed around the block edges to fill voids and also into the foramen space.

This was covered with a resorbable collagen membrane (Ossix, 3i, West Palm Beach, Florida)

Fig. 49 (right) Advanced flap closed without any tension using resorbable sutures. Note the flap design places the vertical incisions at least one tooth distant to the graft area

Figs 50-51 Six months later, the flap is elevated for implant placement. Note residual membrane and layer of soft connective tissue under it, which are then removed along with the fixation screws
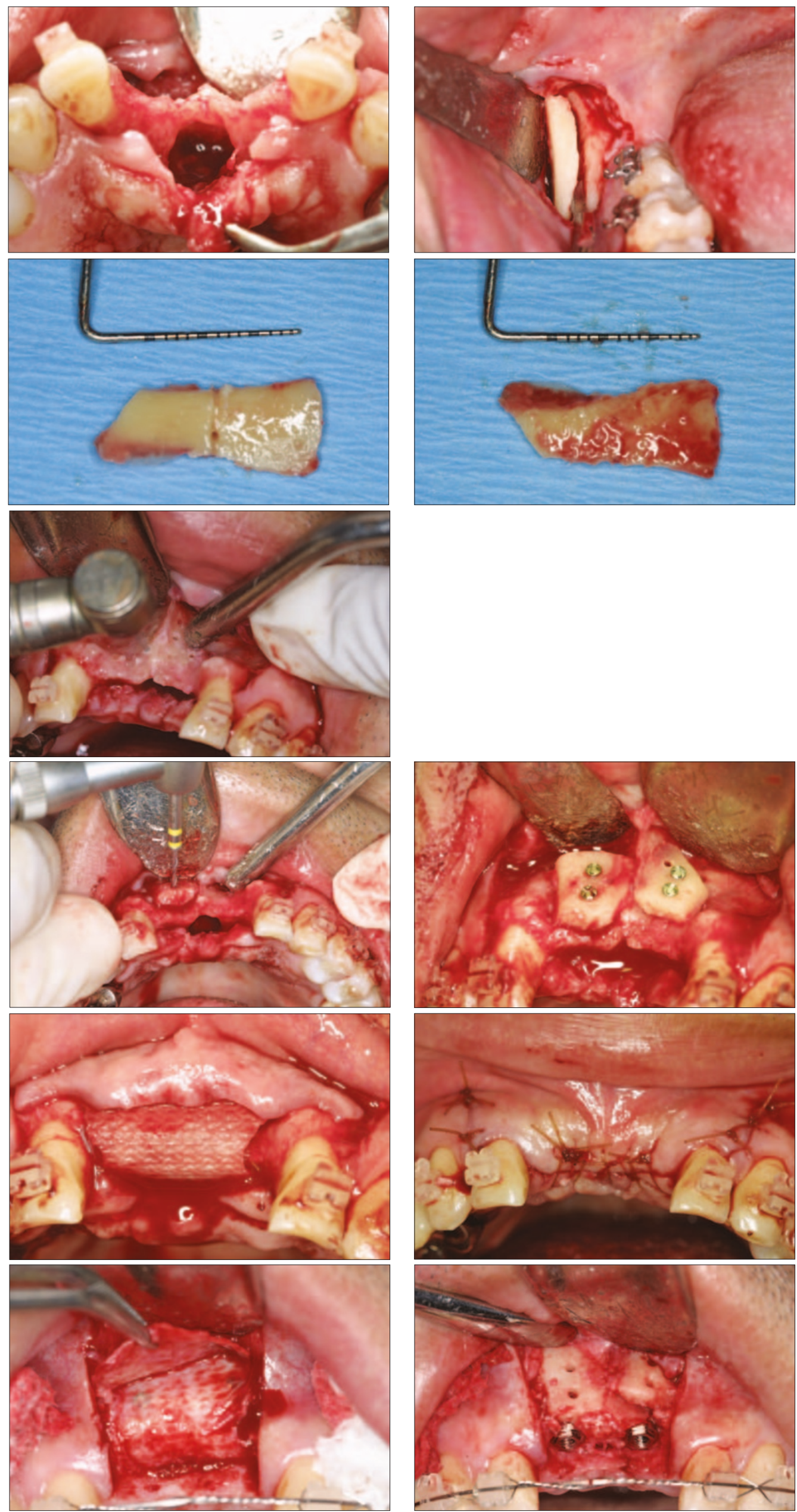

BRITISH DENTAL JOURNAL VOLUME 201 NO. 3 AUG 122006 

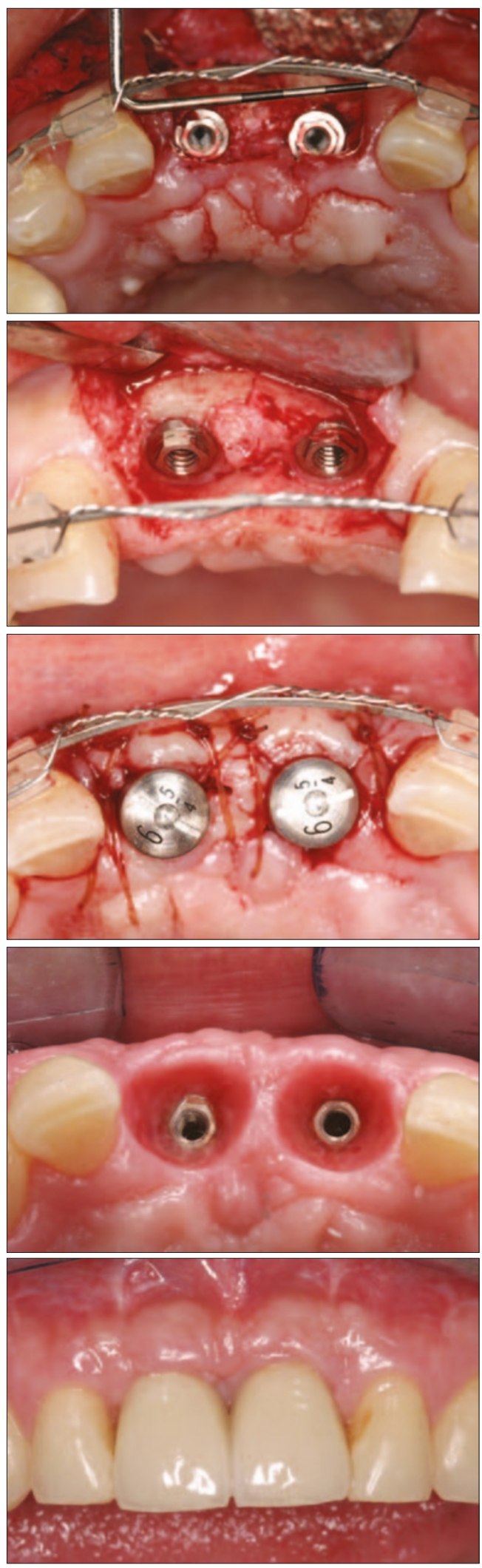
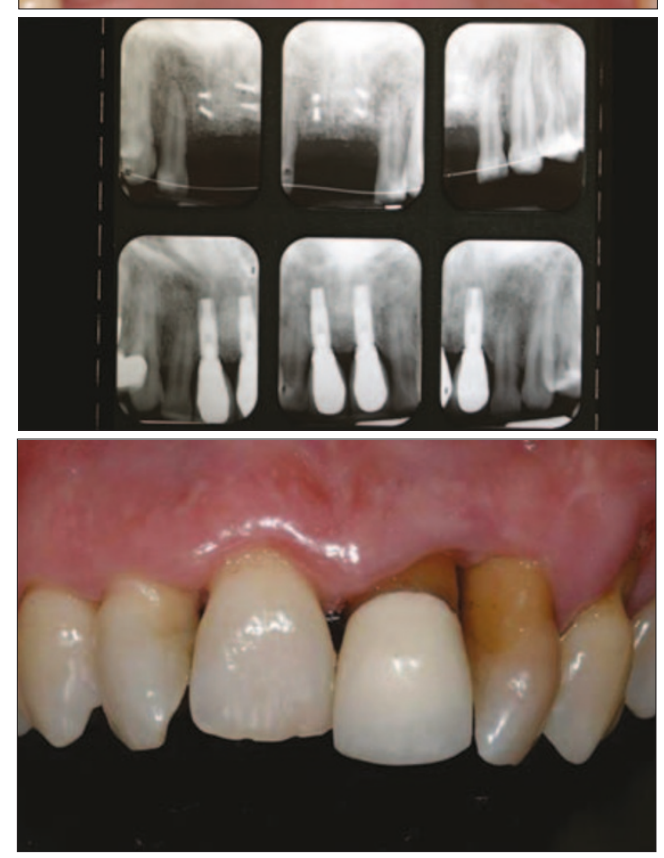

Fig. 52 (left) Occlusal view shows implant fixtures placed, with more than $3 \mathrm{~mm}$ inter-implant distance and slightly countersunk for aesthetic emergence

Fig. 53 (right) Flangeless interim removable appliance
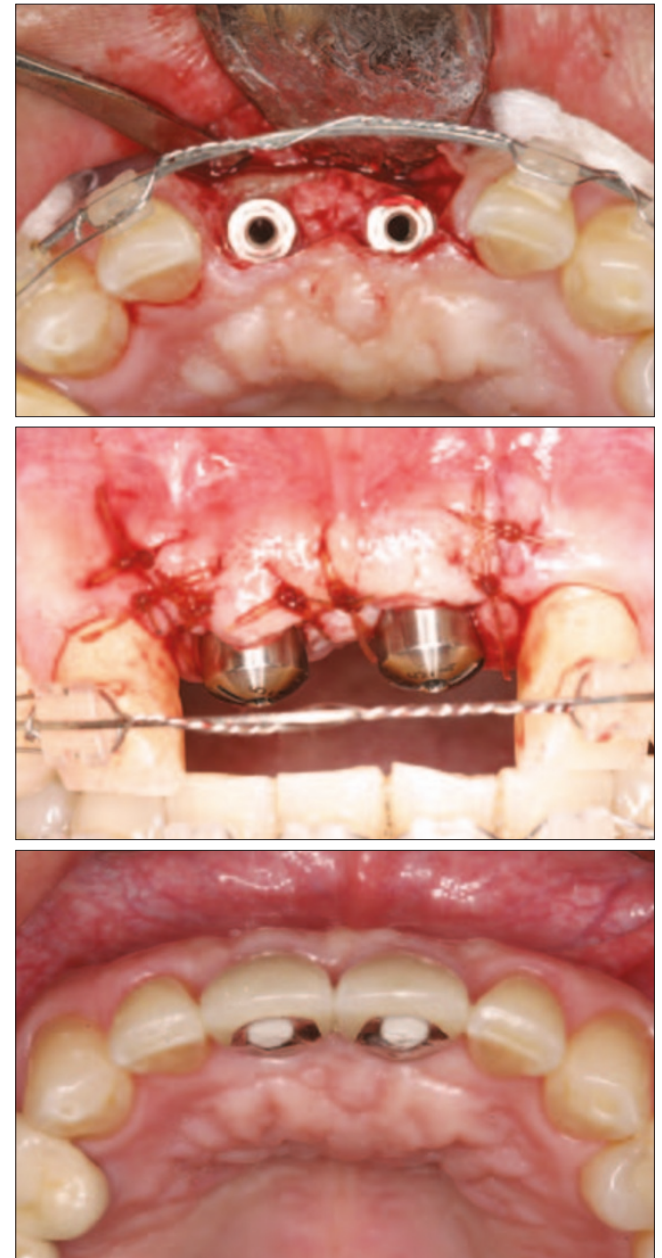

Figs 56-57 Healing abutments placed with sutures

Fig. 58 (left) Several months of loading the implants with a provisional restoration will allow the soft tissue to mature into desired contours prior to placement of final restorations. Note the development of central papilla tissue

Fig. 59 (right) Occlusal view of final restoration. Note the reconstruction of the buccal contours

Fig. 60 (left) Final restorations (Restoration courtesy Dr Seon Ha)

Fig. 61 (right) Radiographs after the bone graft prior to implant placement, and then two years after placement of final restorations. Note stable level of the crestal bone with the interproximal bone supporting the soft tissue architecture

Fig. 62 Initial clinical photo. Patient presents with advanced periodontitis and root resorption of the left maxillary cuspid (Tooth 23 ) 
Figs 63-64 Orthodontic forced eruption at two months and five months to help reduce the bony defect prior to extraction. Orthodontic therapy by Dr Kishibay

Figs 65-67 Radiographs: Initial, during and after movement
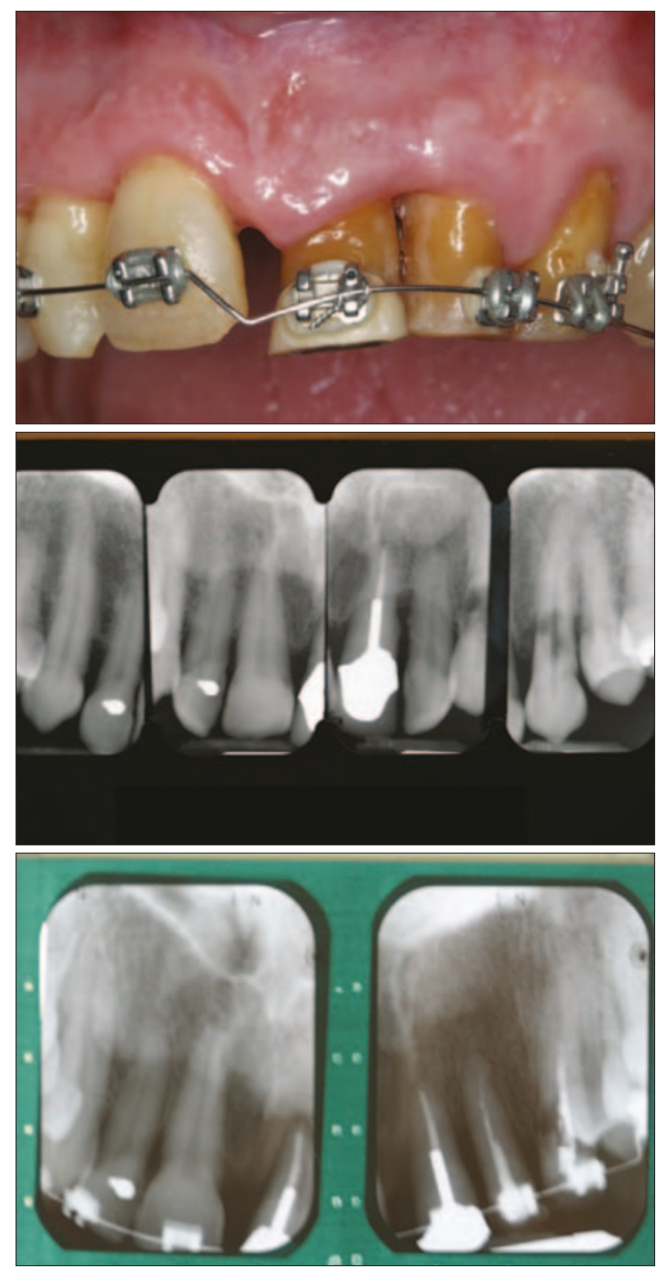

2. Orthodontic forced eruption ${ }^{27}$ to move the gingival complex and crestal bone into a more favourable position prior to extraction (Figs 62-67).

3. Extract and bone graft the socket to help preserve soft tissue contours and minimise collapse of the ridge, if the buccal plate is thin or has a slight dehiscence.

4. Extract and place implant immediately into socket (single rooted teeth and buccal plate is thick and intact).

a. Two stage buried.

b. One stage with healing abutment or customised healing abutment.

c. Immediate load implant with provisional restoration.

5. Extract and wait two to three months, then bone graft (Intra-oral block or GBR). After five to six months healing, place the implant.

If you expect to have advanced ridge resorption subsequent to tooth removal, then staging the case with several procedures is going to be the more predictable option. Extract the tooth and wait two to three months for soft tissue maturity. If it is possible to place the implant in an ideal position, then Gruender ${ }^{28}$ suggests a simultaneous approach; placing the implant with buccal particulate bone graft (suggests Bio-OSS) with a membrane over it, known as Guided Bone Regeneration (GBR). His treatment philosophy for achieving the optimal aesthetic
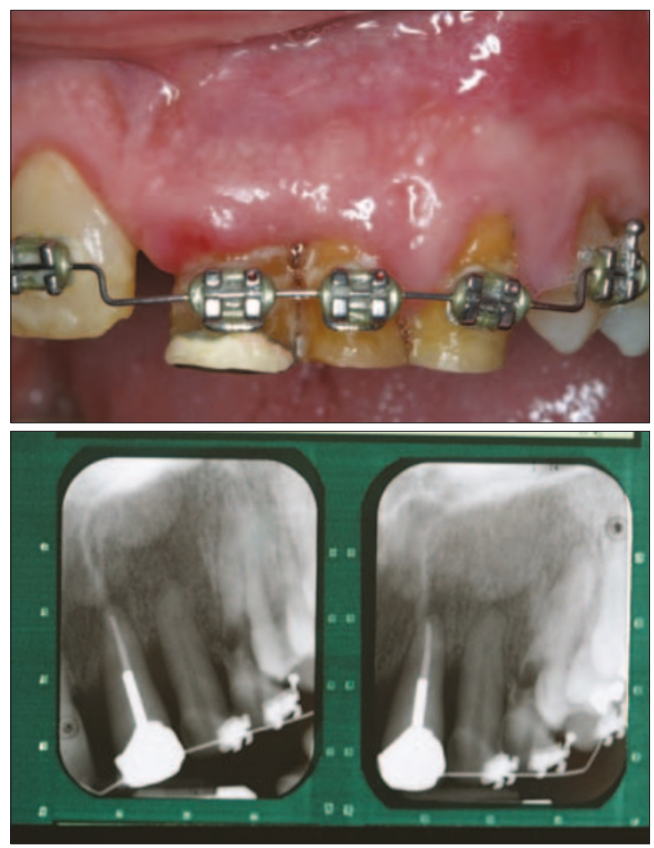

result in the anterior zone requires the site be over-built more than nature provided. Furthermore, during the first year of bone remodelling, crestal resorption down to the first thread of the implant is not restricted only to the interproximal zone as seen on dental radiographs, but also occurs on the buccal aspect and thus affects the supporting hard and soft tissues. This leads to further buccal shrinkage and recession around the implant restoration. Buser ${ }^{29}$ suggests a staged approach to GBR. Build the bone with a block or particulate graft and membrane using fixation screws for stability, and only after six months healing, then place the implant. At that time the options of one stage (exposed healing abutment or immediate loading with a provisional restoration) vs. buried two stage approach can be considered (see clinical case presented in Figs 34-61). If soft tissue grafting is also required, the option to do the soft tissue graft simultaneously with implant placement is possible. Later, it is easier to surgically manipulate these augmented soft tissues at the second stage uncovering procedure.

\section{Timing of loading}

Changing the implant surface from a smooth machined finish to a roughened surface has been shown to improve the rate of bone healing adjacent to the implant. This allows a quicker healing time for osseointegration to be established. With improved and modified drilling techniques, the primary stability of the implant at the time of placement has improved. This has led to the now popular immediate loading with provisional restorations at the time of placement. ${ }^{30}$ At the present time, long term research is not available to show that this will be as predictable as the success rates achieved with the original delayed loading protocol.

The primary factor for success at the time of placement is achieving primary stability. Recent development of the Osstell ${ }^{\mathrm{TM}}$ machine that 
quantifies stability of the fixture using sound resonance $^{31}$ has helped to give the surgeon an objective measurement to determine treatment outcomes. Any micro-motion during the initial phases of bone healing will cause a lack of integration. Failure is most often caused by overloading due to trans-mucosal forces of a removable appliance over the implant site. Any attempt to keep a patient functioning with fixed provisional restorations during the healing phases of treatment, will allow for easier patient management.

Transitional implants ${ }^{32}$ were developed to help support interim fixed or removable prosthesis during the healing phases. These implants have also been used to support a surgical guide and have also been adapted to aid orthodontic treatment, where there is a lack of anchorage.

If immediate loading at the time of final definitive implant placement is to be considered, not only should the initial stability be extremely tight, but control of the occlusion on the provisional interim restoration must be adjusted and monitored carefully through the initial healing period. Single anterior teeth are best for immediate loading as compared posterior teeth where the occlusal forces are far greater. In the fully edentulous cases, immediate loading with a rigid splinted interim prosthesis is advised. ${ }^{33}$

It must be noted that if predictability is desired in the aesthetic zone, treatment options that cut down on treatment time and the number of procedures (extraction and immediate placement with provisional restoration), do not always produce the most reliable aesthetic results. This option increases the risk of failure and also makes it more difficult to predict the future level of the buccal tissue contours, due to shrinkage. It does however, help support the adjacent papilla.

\section{Surgical complications}

In order to minimise post-surgical complications, careful planning of the flap design, and gentle soft tissue manipulation is required. Control of bleeding, along with releasing incisions that allow for flap advancement and closure without tension are mandatory. ${ }^{34}$ Failure to maintain flap coverage due to sloughing or opening of the incision line will lead to delayed healing with compromised results.

Smoking affects both hard and soft tissue healing and has been shown to be the greatest risk factor. ${ }^{35}$

The medically compromised patient ${ }^{36,37}$ (eg Diabetes Mellitus, Autoimmune disease, patients on long term steroids, radiation treatment etc) needs special attention, but is not contra-indicated to receiving treatment. Logical and ethical decisions need to be made with the patient and their physicians.

1. Garber D, Belser U. Restoration driven implant placement with restoration generated site development. Compend Contin Educ Dent 1995; 16: 796-804.
2. Bahat $\mathrm{O}$, Handelsman M. Presurgical treatment planning and surgical guidelines for dental implants. In Wilson T et al. Advanced periodontics. pp 323-340. Chicago: Quintessence.

3. Spear $F$. The challenges of presenting interdisciplinary treatment. Advanced Esthetics Interdiscip Dent 2005; $1: 2$.

4. Becker $B, 0$ chenbein $C$, Tibetts L et al. Alveolar bone anatomic profiles as measured from dry skulls. Clinical ramification. J Clin Periodontol 1997; 24: 727-731.

5. Kois J. Altering gingival levels: The restorative connection. Part 1: Biologic variables. J Esthet Dent 1994; 6: 3-9.

6. Tarnow D P, Magner A W, Fletcher P. The effect of the distance from the contact point to the crest of bone on the presence or absence of the Interproximal dental papilla. $J$ Periodontol 1992; 63: 995-996.

7. Lekholm U, Zarb G A. Patient selection and preparation. In Branemark P I, Albrektsson T, Zarb G A (Eds). Tissueintegrated prostheses: Osseointegration in clinical dentistry. pp 199-209. Chicago: Quintessence, 1985.

8. Sharshak T, Sanders B. Oral anatomy and physiology. In: Preprosthetic oral and maxillofacial surgery. p 15. The CV Mosby Company, 1980.

9. Branemark P I, Albrektsson T, Zarb G A (Eds). Tissueintegrated prostheses: Osseointegration in clinical dentistry. pp 199-209. Chicago: Quintessence, 1985.

10. Buser D, Weber $H$, Bragger $U$, Balsiger $C$. Tissue integration of one-stage ITI implants: 3 -year results of a longitudinal study with hollow-Cylinder and Hollow-Screw Implants. Int J Oral Maxillofac Implants 1991; 6: 405-412.

11. Becker W, Becker B E et al. A prospective multicenter clinical trial comparing one- and two-stage titanium screw shaped fixtures with one-stage plasma sprayed solid-screw fixtures. Clin Implant Dent Relat Res 2000; 2: 159-165.

12. Abrams L. Augmentation of deformed residual edentulous ridge for fixed prosthesis. Compend Contin Educ Gen 1980; $1: 205$.

13. Langer $B, C a l a n g a ~ L$. The subepithelial connective tissue graft. J Prosthet Dent 1980; 44: 363.

14. Garber D A, Rosenberg $E$. The edentulous ridge in fixed prosthodontics. Compend Contin Educ Gen 1981; 2: 212.

15. Palacci $P(E d)$. Esthetic implant dentistry soft and hard tissue management. pp 159-202. Chicago: Quintessence, 2001.

16. Kois J C, Kan J Y K. Predictable peri-implant gingival aesthetics: Surgical and prosthodontic rationales. Prac Proced Aesthet Dent 2001: 13: 691-698.

17. Gruender U, Gracis S, Capelli M. Influence of the 3-d boneto-implant relationship on esthetics. Int J Perio Rest Dent 2005; 25: 113-119.

18. Summers R B. Sinus floor elevation with osteotomes. J Esthet Dent 1998; 10: 164-171.

19. Moy P, Palacci P. Minor bone augmentation procedures. In Palacci P (Ed). Esthetic implant dentistry soft and hard tissue management. pp 137-158. Chicago: Quintessence, 2001.

20. Chin M. Distraction osteogenesis for dental implants. Atlas Oral Maxillofac Surg Clin North Am 1999; 7: 41-63.

21. Hirshfeld A, Wasserman B. A long term survey of tooth loss in 600 treated periodontal patients. J Periodonto/ 1978; 49: 225-237.

22. Boyne $P$, James R A. Grafting of the maxillary sinus with autogenous marrow and bone. J Oral Surg 1980; 38: 613-616.

23. Marx R E, Carlson E R et al. Platelet-rich plasma: growth factor enhancement for bone grafts. Oral Surg Oral Med Oral Pathol Oral Radiol Endod 1998; 85: 638.

24. Jensen 0, Nock D. Inferior alveolar nerve reposition in conjunction with placement of osseointegrated implants: A case report. Oral Surg Oral Med Oral Pathol 1987; 63: 263-268.

25. Buser D, Dula K, Belser U C et al. Localized ridge augmentation using guided bone regeneration. 1. Surgical procedure in the maxilla. Int J Perio Rest Dent 1993; 13: 29-42.

26. Handelsman M, Celletti R et al. Localized Ridge Augmentation: Use of titanium reinforced membranes. J de Paradontologie \&t d'implantologie orale 1997; 16: 243-253.

27. Salama H, Salama M. The role of orthodontic extrusive remodeling in the enhancement of soft and hard tissue profiles prior to implant placement: A systematic approach to the management of extraction site defects. Int J Perio Rest Dent 1993; 13: 312-333.

28. Grunder U. Stability of the mucosal topography around single-tooth implants and adjacent teeth: one year results. Int J Perio Rest Dent 2000; 20: 11-17.

29. Buser $D$, et al. Long term stability of osseointegrated implants in augmented bone: $\mathrm{A} 5$ year prospective study in partially edentulous patients. Int J Perio Rest Dent 2002; 22: 108-117. 
30. Grunder U. Immediate functional loading of immediate implants in edentulous arches: 2 year results. Int J Perio Rest Dent 2001; 21: 545-551.

31. Glauser R, Sennerby L, Meredith N et al. Resonance frequency analysis of implants subjected to immediate or early functional occlusal loading. Successful vs. failing implants. Clin Oral Implants Res 2004; 15: 428-434.

32. Froum S, Emtiaz S, Bloom M J et al. The use of transitional implants for immediate fixed temporary prostheses in cases of implant restorations. Prac Perio Aesthet Dent 1998; 10 737-746.

33. Tarnow D P, Emitiaz S, Classi A. Immediate loading of threaded implants at stage one surgery in edentulous arches: ten consecutive case reports with $1-5$ years data. Int J Oral Maxillofac Implants 1997; 12: 319-324.

34. Bahat 0 , Handelsman M. Periodontal reconstructive flaps - Classification and surgical considerations. Int J Perio Rest Dent 1991; 11: 481-487.

35. Bain C, Moy P. The association between the failure of dental implants and cigarette smoking. Int J Oral Maxillofac Implants 1993; 8: 609-615.

36. Moy P etal. Dental implant failure rates and associated risk factors. Int J Oral Maxillofac Implants 2005; 20: 569-577.

37. Marder M Z. Medical conditions affecting the success of dental implants. Comped Contin Ed Dent 2004; 25: 739-771. 